\title{
e-Migrinter
}

15 | 2017

Migrinter a trente ans : analyses et portraits

\section{Migrinter a 30 ans. Analyses et portraits}

Marine Bertrand, Olivier Clochard, Julie Lemoux, Mélanie Pénicaud et Sarah Przybyl

\section{OpenEdition}

Journals

Édition électronique

URL : https://journals.openedition.org/e-migrinter/847

DOI : $10.4000 /$ e-migrinter.847

ISSN : 1961-9685

Éditeur

UMR 7301 - Migrinter

Référence électronique

Marine Bertrand, Olivier Clochard, Julie Lemoux, Mélanie Pénicaud et Sarah Przybyl, « Migrinter a 30 ans. Analyses et portraits », e-Migrinter [En ligne], 15 | 2017, mis en ligne le, consulté le 20 mai 2021. URL : http://journals.openedition.org/e-migrinter/847 ; DOI : https://doi.org/10.4000/e-migrinter.847

Ce document a été généré automatiquement le 20 mai 2021.

Tous droits réservés 


\title{
Migrinter a 30 ans. Analyses et portraits
}

\author{
Marine Bertrand, Olivier Clochard, Julie Lemoux, Mélanie Pénicaud et \\ Sarah Przybyl
}

1 Ce numéro spécial, coordonné par l'équipe de rédaction de la revue e.Migrinter, marque le $30^{\text {ème }}$ anniversaire du laboratoire Migrinter. L'unité de recherche a été fondée en 1985 par Gildas Simon dans l'objectif de regrouper des activités de recherche, de formation, de publication et de documentation structurées autour de l'objet des migrations internationales, des liens avec les sociétés d'origine et des relations interethniques. Face à la complexification croissante des dynamiques migratoires et aux multiples enjeux créés par leurs interactions avec les espaces et les sociétés contemporaines, cet objet de recherche est plus que jamais d'actualité, encourageant une diversité d'approches permise par la structuration pluridisciplinaire du laboratoire.

2 Le numéro anniversaire proposé ici est composé de treize articles et billets. Nous avons proposé à des chercheurs de revenir sur des thématiques, espaces de réflexion ou temps forts structurant la recherche en migrations internationales et en particulier à Migrinter. Le dossier de ce numéro s'organise exceptionnellement en deux parties. La première, intitulée "Revisiter les fondamentaux des migrations internationales », vise à offrir un regard intergénérationnel sur quatre objets d'études ou notions travaillés à Migrinter et dans le champ plus large de la recherche en migrations internationales. La seconde partie de ce dossier, intitulée "Enseigner les migrations internationales » propose un retour historique et une réflexion pédagogique autour de l'enseignement des migrations internationales, depuis l'expérience du Master Migrations internationales, espaces et société de l'Université de Poitiers. Cette réflexion est menée par des responsables de formations sur les migrations internationales mais aussi au travers d'un retour d'expérience d'une ancienne étudiante.

3 Faisant suite au dossier, une deuxième partie intitulée «Les coulisses de la Vie du Labo » vient compléter ce numéro. Sarah Przybyl et Julie Lemoux y proposent une série de courts articles sur l'expérience quotidienne de la recherche de plusieurs acteurs du 
laboratoire Migrinter. Documentaliste et secrétaire d'e-migrinter, secrétaire de rédaction de la Revue européenne des Migrations internationales (REMI), gestionnaire CNRS, géomaticienne : ces personnalités du laboratoire y présentent leurs activités et nous offrent l'occasion de découvrir la richesse de la recherche à Migrinter à partir du point de vue d'autres acteurs de la recherche. Un article associé à une vidéo prolonge ces portraits en présentant le "Réseau Migrations " créé à Poitiers en juin 2014 à l'occasion d'une première journée d'étude rassemblant des jeunes chercheurs en migrations internationales. Enfin, un dernier article présente l'exposition "Démineurs", initiée par Fred Soupa (réalisateur et membre de l'association La colline) et Sarah Przybyl (géographe à Migrinter), sur le thème des mineurs isolés étrangers et mise en place en marge du colloque anniversaire des 30 ans du laboratoire et ouvert au grand public.

4 Les quatre premiers articles du dossier mettent en dialogue plusieurs générations de chercheurs sur des thématiques centrales dans le champ des migrations internationales. Les auteurs proposent de revisiter des textes fondateurs, tant en sociologie qu'en géographie.

5 L'article de Julie Garnier revient sur la sociologie des relations interethniques en milieu urbain développée par Véronique de Rudder et Michelle Guillon. À la (re)lecture d'un texte de 1986 qui analyse les situations résidentielles comme des situations de " cohabitations interethniques », Julie Garnier montre les apports fondamentaux de ces auteures pour contribuer à l'émergence de questions de recherche qui dépassent le "problème de l'immigration", favoriser une compréhension relationnelle et dynamique des situations sociales observées, tout en favorisant des approches pluridisciplinaires aux dispositifs méthodologiques novateurs.

6 Thomas Lacroix et Julie Lemoux discutent la notion de "génération d'émigrés » introduite par Abdelmalek Sayad en 1977, et interrogent cet outil conceptuel à la lumière des évolutions qui structurent le champ migratoire algérien depuis les années 2000. Les processus d'élargissement de l'espace migratoire algérien et de diversification des profils des personnes liées à ces déplacements conduisent à questionner l'idée d'un "quatrième âge» de l'émigration algérienne. L'article revient d'abord sur la conception des trajectoires d'émigrés, développées par Abdelmayek Sayad et telles que pensées en termes de "générations ». Puis, il s'agit de montrer dans quelle mesure on peut parler de nouvel âge de l'émigration algérienne à partir des années 2000, mais aussi comment l'approche en termes de génération devient peu à peu insuffisante pour penser la dynamique actuelle de ces parcours migratoires.

7 L'article d'Iris Polyzos présente et commente un entretien réalisé avec Emmanuel Ma Mung sur l'articulation des notions d'autonomie et de projet migratoire. Celles-ci sont interrogées au regard du parcours scientifique du géographe, jalonné par une succession d'objets de recherche - l'évolution des sociétés agraires en Tunisie, le commerce ethnique en France, les diasporas, etc. -, qui ont conduit le géographe à mobiliser, repenser et façonner ces notions. Au-delà des positionnements théoriques et conceptuels adoptés par Emmanuel Ma Mung, l'article rend compte de la dynamique du chercheur qui souhaite maintenir ces notions ouvertes et leur caractère évolutif en fonction des objets de recherche.

$8 \quad$ L'article de Nelly Robin revient sur près de deux décennies de recherche au Maghreb et dans plusieurs pays de l'Afrique subsaharienne où elle a conduit des terrains d'enquête en zone de conflits comme dans la région casamançaise et dans le monde judiciaire 
sénégalais. La géographe discute l'évolution de la posture du chercheur inscrit dans son terrain sur le temps long. Elle interroge ici le parallèle entre ses objets de recherche et son itinéraire scientifique, ainsi que la construction de sa posture sur des terrains qu'elle qualifie de « minés ».

9 La seconde partie du dossier, qui alimente une réflexion sur la question « comment enseigner les migrations internationales? ", est composée dans un premier temps d'un article rédigé par David Lessault, Adelina Miranda et Véronique Lassailly-Jacob. Cette question fit l'objet d'un atelier lors du colloque des trente ans « Penser les migrations pour repenser la société » qui s'est tenu à l'Université de Poitiers du 21 au 24 juin 2016. Aussi, cet article propose-t-il une synthèse des propos tenus alors par Véronique Lassailly-Jacob, Pierre Kamdem et Papa Sakho, articulés par les réflexions des auteurs, responsables à différentes époques du master en migrations internationales proposé à Migrinter. En partant de l'histoire de ce master, de ses fondements théoriques et de ses grandes évolutions pédagogiques, puis en interrogeant les défis de l'internationalisation de l'enseignement des migrations internationales, les auteurs se penchent sur les enjeux de la transmission des savoirs et des compétences autour de ce champ de recherche. Ces enjeux sont de taille dans le contexte actuel d'intense production d'informations sur les migrations, portée par les médias, les politiques, les chercheurs et le monde associatif.

Cette partie est alimentée par un billet d'Alba Otero Gracia qui met en perspective son expérience au sein du Master Migrations internationales à l'Université de Poitiers à partir du récit d'une anecdote vécue à l'occasion d'un cours. Partant d'une question relative à la définition de la famille, Alba Otero Gracia montre, à travers le débat suscité entre les étudiants, comment la question migratoire ne peut être comprise sans la relier aux dynamiques sociétales. Elle raconte comment les questions liées aux migrations l'ont plus largement conduite à repenser les sociétés dans leur diversité et à façonner son esprit critique face aux multiples constructions de pensée qui circulent autour de l'objet migration.

11 La rubrique «Les coulisses de la vie du labo » s'est tournée vers les acteurs qui en sont au cœur. Emmanuel Demaury, Carole Tardif, Audrey Brosset et Nelly Martin partagent ici leur expérience professionnelle dans cet espace de recherche et d'enseignement. Ceux dont l'activité est souvent moins bien connue que celle des chercheurs, et pourtant essentielle au bon fonctionnement d'un laboratoire, dévoilent ici leur quotidien en quatre portraits.

12 Le billet de Carole Tardif décrit son activité de technicienne en documentation et en Publication assistée par Ordinateur (PAO). Parmi ses missions de gestion du fond documentaire du centre de documentation de la MSHS et de valorisation de la recherche, elle détient les compétences techniques de publication de la revue $e^{-}$ migrinter.

13 Le texte rédigé par Emmanuel Demaury concerne sa mission de gestion administrative et financière de l'Unité mixte de recherche (UMR). Attaché au bureau d'administration de l'UMR Migrinter, il relate sa journée-type, plongeant ici le lecteur dans le quotidien des relations entre l'équipe de recherche et l'équipe administrative.

14 Nelly Martin, ingénieur d'études et géomaticienne, revient quant à elle sur le parcours qui l'a conduite à rejoindre l'équipe. Récemment arrivée dans le laboratoire, elle décrit sa mission qui consiste à produire des cartes en utilisant des Systèmes d'Informations 
géographiques (SIG), compétence qu'elle met en œuvre dans plusieurs programmes de recherche et qu'elle transmet aux étudiants du Master.

À son tour, Audrey Brosset, secrétaire de rédaction de la Revue européenne des Migrations internationales (REMI) de l'Université de Poitiers, décrit ses missions dans la chaîne éditoriale de la revue. Au cœur de ses activités quotidiennes, Audrey Brosset propose au lecteur une plongée dans le fonctionnement de la revue et relate ses expériences auprès des chercheurs ayant eu pour mission la direction scientifique de la revue.

Par ailleurs, Josepha Milazzo, Ewa Tartakowsky et Jordan Pinel, du Réseau Migration, présentent la structuration et les objectifs du réseau rassemblant des jeunes chercheurs de tous horizons et de toutes disciplines autour du thème des migrations internationales. Le réseau et ses activités sont présentés à travers une vidéo accompagnée d'un texte de Celio Sierra-Paycha, doctorant au laboratoire Migrinter, qui raconte la manière dont les membres ont voulu mettre en images la constitution du réseau à travers la réalisation d'une infographie.

Enfin, un article de Sarah Przybyl clôture ce numéro dans lequel elle présente le projet "Démineurs ». Au cours de sa recherche doctorale sur les parcours migratoires et de protection des mineurs isolés étrangers, Sarah Przybyl a souhaité mobiliser l'approche audiovisuelle comme outil d'enquête. Après avoir soutenu sa thèse en décembre 2016, elle profite de la publication de ce numéro pour revenir sur les contours du projet "Démineurs ", co-construit avec Fred Soupa et sur la production d'une exposition à partir des court-métrages.

\section{AUTEURS}

\section{MARINE BERTRAND}

Doctorante en anthropologie, Migrinter - UMR 7301, CNRS / Université de Poitiers

marine.bertrand@univ-poitiers.fr

\section{OLIVIER CLOCHARD}

Chargé de recherche au CNRS, Migrinter - UMR 7301 CNRS / Université de Poitiers olivier.clochard@univ-poitiers.fr

\section{JULIE LEMOUX}

Doctorante en sociologie, Migrinter - UMR 7301 CNRS / Université de Poitiers julie.lemoux@gmail.com

\section{MÉLANIE PÉNICAUD}

Docteure en anthropologie, Migrinter - UMR 7301 CNRS / Université de Poitiers melaniepenicaud@gmail.com 
SARAH PRZYBYL

Docteure en géographie, Migrinter - UMR 7301 CNRS / Université de Poitiers sarah.przybyl@gmail.com 